Review began 09/30/2021 Review ended 11/03/2021 Published 11/09/2021

๑) Copyright 2021

Rodrigues et al. This is an open access article distributed under the terms of the Creative Commons Attribution License CCBY 4.0., which permits unrestricted use, distribution, and reproduction in any medium, provided the original author and source are credited.

\section{An Atypical Pleomorphic Lipomatous Tumor Presenting As Groin Mass}

\author{
Egon Rodrigues $^{1}$, Florinda Cardoso ${ }^{1}$, Horácio Scigliano ${ }^{2}$, Mário Nora ${ }^{1}$ \\ 1. General Surgery, Centro Hospitalar de Entre Douro e Vouga, Santa Maria da Feira, PRT 2. Anatomical Pathology \\ Laboratory Dr. Albino Oliveira (Unilabs), Centro Hospitalar de Entre Douro e Vouga, Santa Maria da Feira, PRT \\ Corresponding author: Egon Rodrigues, egon.rodrigues9@gmail.com
}

\begin{abstract}
Cutaneous tumors with adipocyte differentiation are frequently excised by surgeons in their daily clinical practice and sometimes less common histological diagnoses arise. Knowledge of different pathological entities and their natural history is essential for better patient management.
\end{abstract}

Atypical spindle cell/Pleomorphic lipomatous tumor (ASPLT) is a recent group included in the WHO classification. We report a case of a middle-aged man with an atypical pleomorphic lipomatous tumor in an unusual location.

Categories: Pathology, General Surgery

Keywords: soft-tissue tumor, groin mass, adipocytic tumor, atypical spindle cell/pleomorphic lipomatous tumor, atypical pleomorphic lipomatous tumor

\section{Introduction}

Adipocytic tumors are the most common soft-tissue tumors, and surgical removal of these lesions is a procedure widely performed in general surgery. In 2020, WHO published a new classification of Soft Tissue and Bone Tumours, differentiating several entities [1]. The atypical spindle cell/pleomorphic lipomatous tumor (ASPLT) was included as an entity for the first time in this edition. Although it is considered a rare subtype $[2,3]$, its incidence has not yet been described.

We report the case of an atypical pleomorphic lipomatous tumor that presented as a rapidly enlarging mass in the right groin region.

\section{Case Presentation}

A 58-year-old man, with no relevant medical history, presented with a history of an enlarging painless mass at his right groin region for the past three months (Figure 1). The patient did not have any other complaints or symptoms. Physical examination revealed a firm, skin-colored and mobile tumor with well-defined margins ( $5 \mathrm{~cm}$ largest diameter). There were no palpable adenomegalies. 


\section{Cureus}

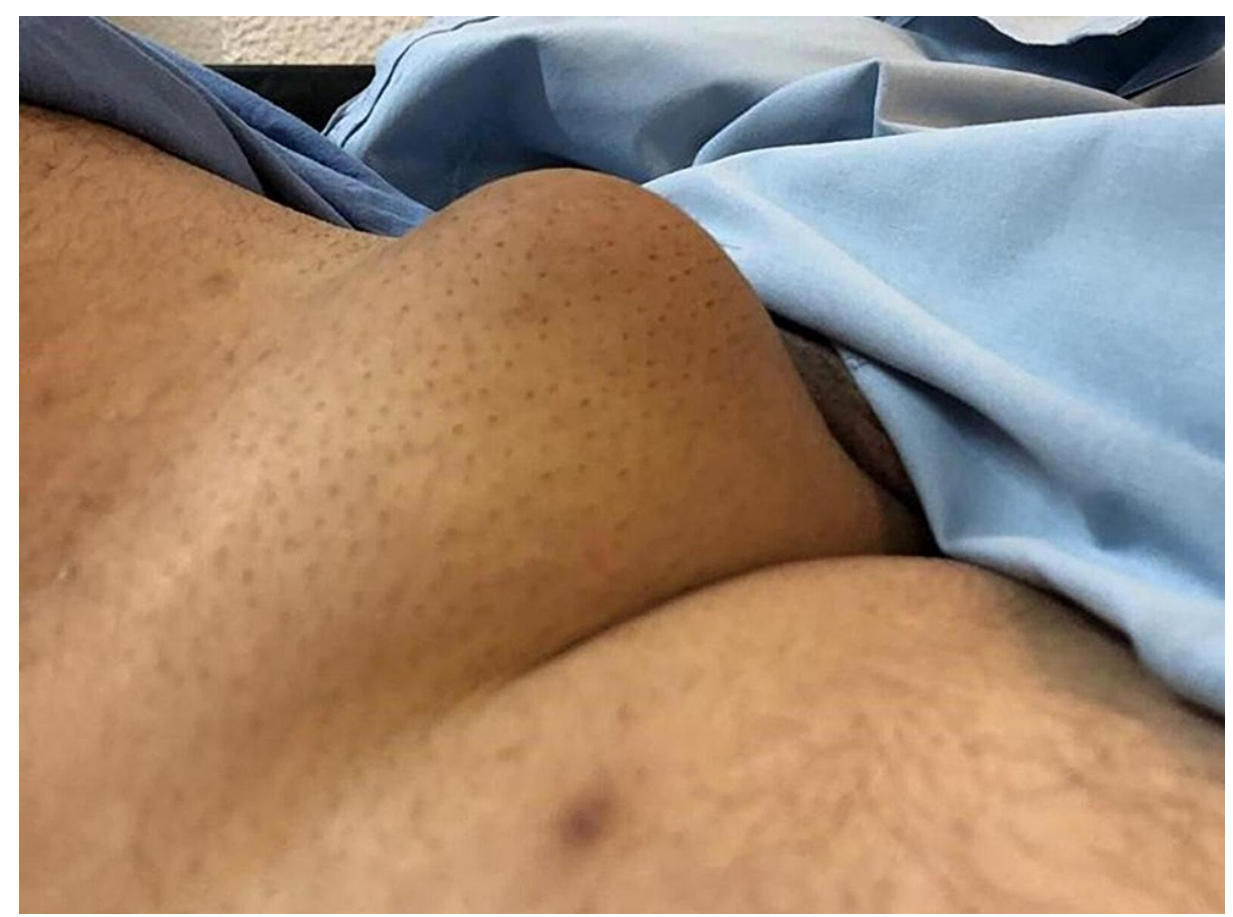

\section{FIGURE 1: Mass in the right groin region.}

The patient was referred to the General Surgery department by a urologist, with suspicion of a soft-tissue tumor. An MRI described a "focal subcutaneous lesion with nodular morphology of $4.7 \mathrm{~cm}$ and no malignancy features". Based on clinical and image findings, it was decided to perform an excisional biopsy.

Despite the apparent benign characteristics, the lesion was surgically removed along with the surrounding adipose tissue, preserving the margins. There were no complications related to the procedure.

Grossly, it was a subcutaneous nodular non-capsulated solid lesion, multilobulated, well-circumscribed, greyish-yellowish, without necrotic areas (Figure 2). Microscopically, a variable amount of atypical bland spindle cells and mature adipocytes were seen, with multinucleated floret-like cells in a myxoid stroma with ropey collagen bundle cells. Sclerosing areas were not disclosed (Figure 3). On immunohistochemistry, the tumor was stained for CD34, S100, and MDM2 (focal-weak), whereas CDK4 expression was absent (Figure 4). Based on these findings, an atypical pleomorphic lipomatous tumor was diagnosed.

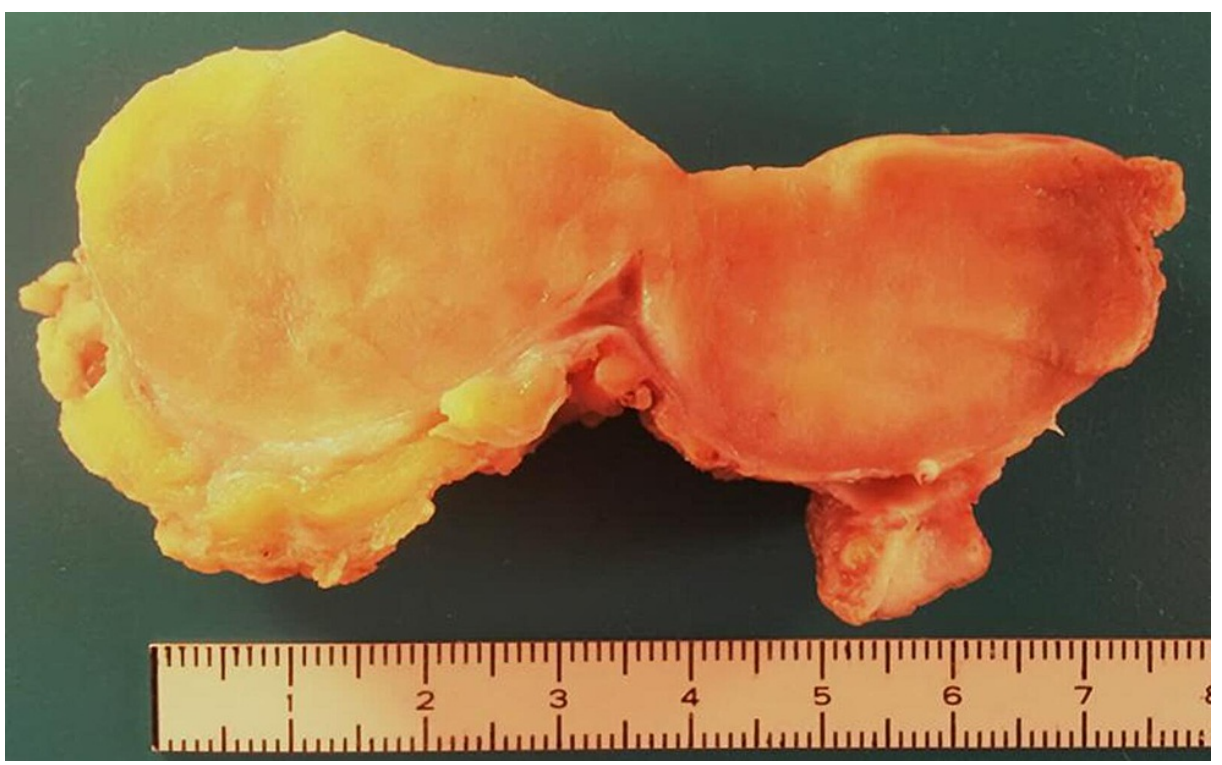

FIGURE 2: Gross aspect of the tumor. 


\section{Cureus}

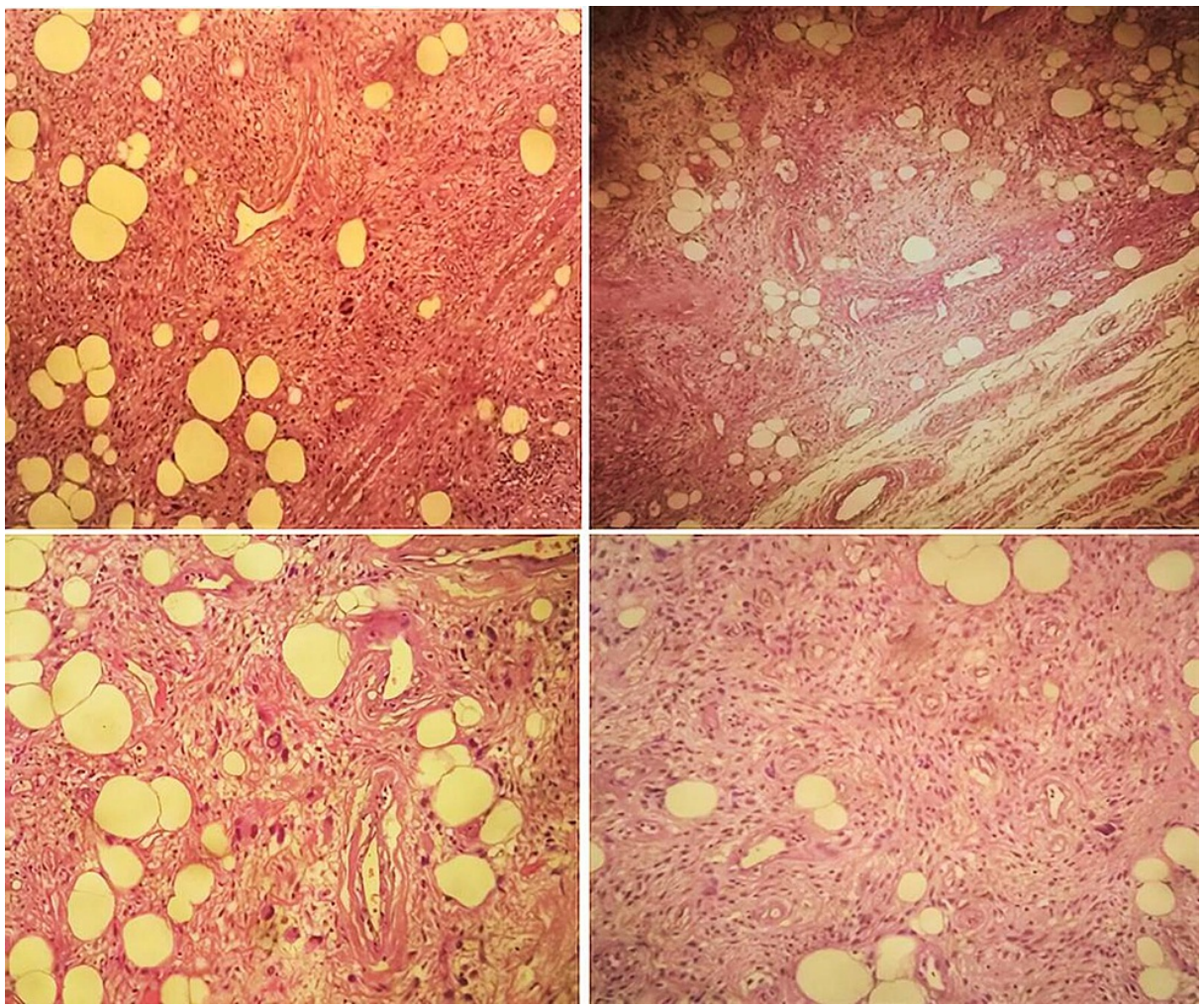

FIGURE 3: H\&E stain.
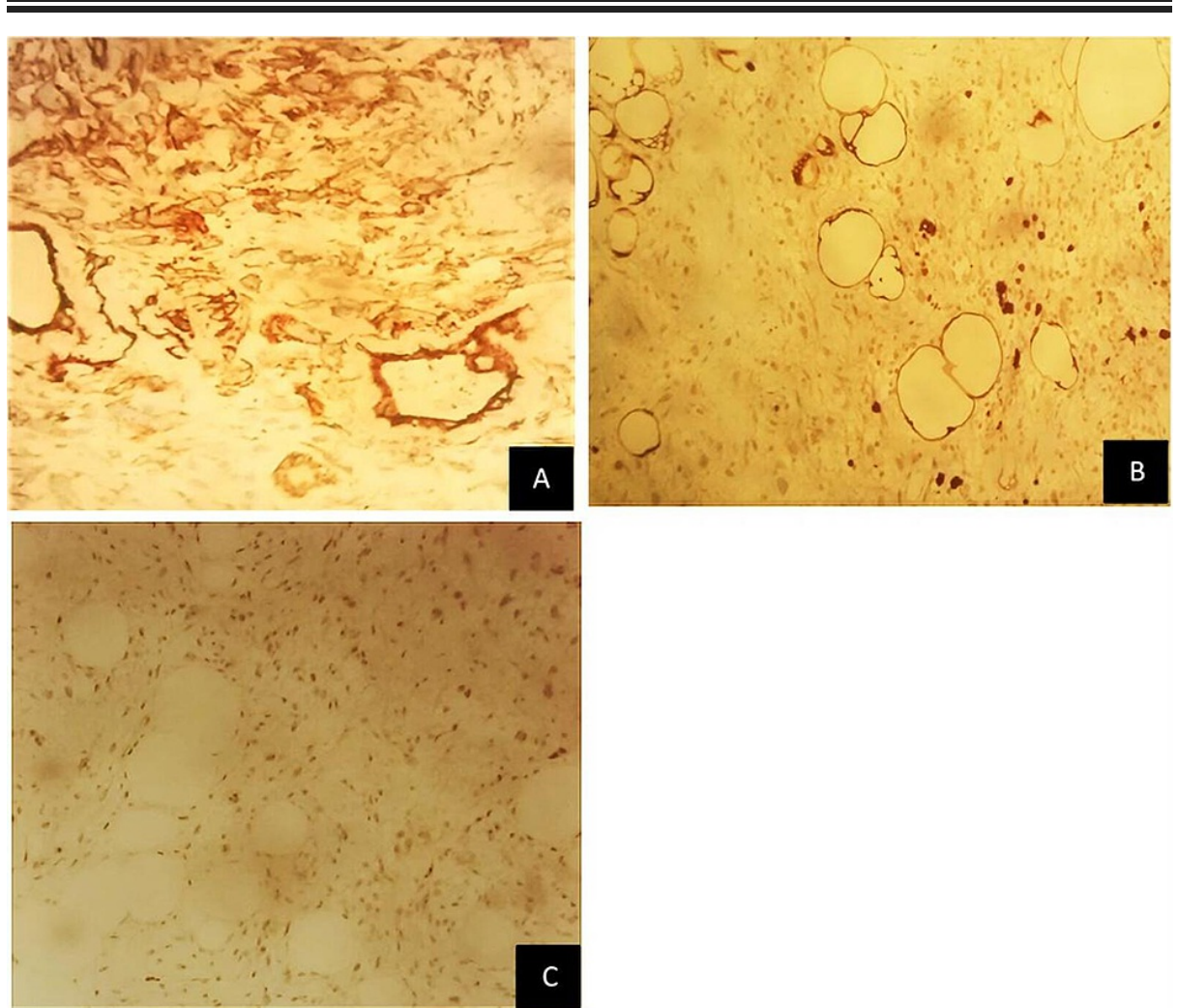

FIGURE 4: Immunocytochemistry of (A) CD34; (B) S100; and (C) MDM2.

\section{Discussion}

Cutaneous tumors with adipocyte differentiation are frequently excised by surgeons. Although they are 
mostly lipomas or angiolipomas, other rare entities can arise and raise doubts. Recently, WHO published the $5^{\text {th }}$ edition of soft-tissue tumors classification which included a new group, the atypical spindle cell/pleomorphic lipomatous tumor (ASPLT) [1].

Mentzel T et al. proposed the term “atypical spindle cell lipoma” for the first time in 2010 [4], suggesting that these neoplasms most likely represent an independent entity closely related to spindle cell lipoma rather than a morphologic variant of atypical lipomatous tumor/well-differentiated liposarcoma [4]. Since then, other works have given strength to this theory [2,5-7], representing now a newly established diagnostic entity [1].

ASPLT is a morphologic spectrum of benign adipocytic neoplasms [1]. The cellularity of the tumors is the basis of the spectrum. At one end, tumors can be paucicellular with few atypical spindle cells and abundant extracellular matrix. At the other extreme, they may be significantly more cellular, with less extracellular matrix [8]. As result, there is a wide range of microscopic appearance of ASPLT due to the extremely variable proportions of spindle cells, adipocytes, lipoblasts, and extracellular matrix [1]. Our case represents an atypical pleomorphic lipomatous tumor characterized by the presence of floret-like multinucleated giant cells [7].

This entity can affect both sexes, with a slight male predominance, including mostly middle-aged adults with a peak in the sixth decade [1], likewise our patient. Unlike classic spindle cell/pleomorphic lipomas, ASPLT has a wide anatomical distribution [7] and the clinical differential diagnosis depends on the involved location. The most common locations are the extremities [1]. Abdominal wall and groin areas are infrequent locations. Literature search [2-7, 9-16] shows that Bahadır B et al. [2] and Anderson WJ et al. [9] each described a case in the groin region. Also, two more cases were described on the abdominal wall [2] and Mariño-Enriquez A et al. [6] described 15 cases on the trunk, not specifying the affected regions.

For palpable lesions, ultrasound can be used in the initial investigation, but MRI remains the imaging gold standard [17]. Our patient already had an MRI report, so further diagnostic imaging evaluation was not necessary. Although the MRI favored the presence of a benign soft-tissue tumor, the rapid growth and size raised concerns about its etiology. Johnson CJ et al. found that tumor depth, a size larger than $5 \mathrm{~cm}$, and history of rapid growth were the most sensitive markers of malignancy [18]. When technically possible and available, a core needle biopsy can be performed for histological examination, particularly if a sarcoma is the main suspicion [19]. However, it is not mandatory, especially when tumors are superficial. In this case, we chose to perform an excisional biopsy given the easy access and since our main suspicion was a benign tumor. Nevertheless, the lesion was removed with gross margins preserved, as it did not cause greater aesthetic or functional damage. Frozen section diagnosis and fine-needle aspiration are generally not recommended.

Histological differential diagnosis of ASPLT is highly dependent on the predominant histological features of each tumor. This entity can be considered a category between classic spindle cell/pleomorphic lipoma and liposarcoma, namely: WDL (also designated atypical lipomatous tumors) or pleomorphic liposarcoma (PLS) in cases with significant pleomorphism [2]. In dubious situations, immunohistochemistry and/or molecular tests can be carried out. In ASPLT, the neoplastic cells frequently express CD34, S100, and desmin, whereas MDM2 and CDK4 expression are rare. Expression of MDM2 or CDK4 can be seen in 5\% of cases, but always in the absence of corresponding gene amplification. Simultaneous MDM2 and CDK4 expression has never been described [8]. In the present case, the tumor was stained for CD34 and S100 (desmin was not evaluated). There was also a focal and weak expression of MDM2, while CDK4 expression is absent.

Regarding the differential diagnosis of malignancy, PLS can involve the dermis or subcutaneous tissue in $25 \%$ of cases. Most patients report a rapidly growing painless mass (median: 3-6 months) [1], similar to our case. However, it is characterized by a large size, greater pleomorphism, high mitotic activity, and tumor necrosis. Our findings, such as floret-like multinucleated cells, are typical of ASPLT instead of PLS [6]. WDL is a locally aggressive tumor with a risk of dedifferentiation, which appears most often in the proximal extremities and the retroperitoneum [1]. Terminology of atypical lipomatous tumor or WDL primary depends on tumor's location and relates to its resectability [8]. These tumors affect adults within the same age range as ASPLT. They may be morphologically indistinguishable from fat-rich ASPLT but can be distinguished by MDM2/CDK4 immunohistochemistry or by molecular testing for MDM2 gene amplification [8].

Regarding benign differential diagnosis, classic spindle cell/pleomorphic lipoma are capsulated lesions with no atypia [6], that are immunohistochemical similar to ASPLT. The distinction between this entity and an ASPLT with focal atypia can be extremely difficult. If the lesion appears on the posterior neck and shoulder, then classic spindle cell/pleomorphic lipoma is most likely [8]. Unlike ASPLT, the recurrence rate is low [1]. Other differential diagnoses are angiofibroma and myofibroblastoma, nodular CD34+ neoplasms like ASPLT, which have an anatomical preference for the groin area. In general, these two entities show a less-prominent fat component, mainly consisting of spindle cells with a hyalinized stromal background [20].

Complete surgical excision (i.e., lesion-free margin) is the appropriate treatment for ASPLT. Incompletely removed lesions may locally recur in $10-15 \%$ of the cases [1]. In cases of incomplete excision, widening of 
the margins or patient follow-up for a possible local recurrence seem suitable options. There is no need for regular imaging control. To the best of our knowledge, there is no risk for metastasis [1], hence there is no role for adjuvant treatment.

\section{Conclusions}

When a soft-tissue tumor is suspected, a diagnostic approach may include an ultrasound or an MRI and eventually a core needle biopsy. The diagnosis of ASPLT can be challenging and the morphological differential diagnostic range can be broad due to the wide variety of microscopic appearances. Recognition of morphologic clues and immunohistochemistry/molecular tests to confirm the diagnosis and exclude WDL and PLS are crucial. Although it is a benign adipocytic tumor, it carries a considerable risk of local recurrence.

\section{Additional Information \\ Disclosures}

Human subjects: Consent was obtained or waived by all participants in this study. Conflicts of interest: In compliance with the ICMJE uniform disclosure form, all authors declare the following: Payment/services info: All authors have declared that no financial support was received from any organization for the submitted work. Financial relationships: All authors have declared that they have no financial relationships at present or within the previous three years with any organizations that might have an interest in the submitted work. Other relationships: All authors have declared that there are no other relationships or activities that could appear to have influenced the submitted work.

\section{References}

1. WHO Classification of Tumours Editorial Board: Soft Tissue and Bone Tumours. IARC, Lyon (France); 2020.

2. Bahadır B, Behzatoğlu K, Hacıhasanoğlu E, Koca SB, Sığırcı BB, Tokat F: Atypical spindle cell/pleomorphic lipomatous tumor: a clinicopathologic, immunohistochemical, and molecular study of 20 cases. Pathol Int. 2018, 68:550-556. 10.1111/pin.12719

3. Zhang Z, Liu N, Chen M, Peng R, Chen HJ, Zhang HY: Atypical spindle cell lipomatous tumor/atypical pleomorphic lipomatous tumor and atypical mammary-type myofibroblastoma: clinicopathological, immunohistochemical, and multiplex fluorescence in situ hybridization analyses. Chin Med J (Engl). 2021, 134:2370-2372. 10.1097/CM9.0000000000001692

4. Mentzel T, Palmedo G, Kuhnen C: Well-differentiated spindle cell liposarcoma ('atypical spindle cell lipomatous tumor') does not belong to the spectrum of atypical lipomatous tumor but has a close relationship to spindle cell lipoma: clinicopathologic, immunohistochemical, and molecular analysis of six cases. Mod Pathol. 2010, 23:729-736. 10.1038/modpathol.2010.66

5. Creytens D, van Gorp J, Savola S, Ferdinande L, Mentzel T, Libbrecht L: Atypical spindle cell lipoma: a clinicopathologic, immunohistochemical, and molecular study emphasizing its relationship to classical spindle cell lipoma. Virchows Arch. 2014, 465:97-108. 10.1007/s00428-014-1568-8

6. Mariño-Enriquez A, Nascimento AF, Ligon AH, Liang C, Fletcher CD: Atypical spindle cell lipomatous tumor: clinicopathologic characterization of 232 cases demonstrating a morphologic spectrum. Am J Surg Pathol. 2017, 41:234-244. 10.1097/PAS.0000000000000770

7. Creytens D, Mentzel T, Ferdinande L, et al.: "Atypical" pleomorphic lipomatous tumor: a clinicopathologic, immunohistochemical and molecular study of 21 cases, emphasizing its relationship to atypical spindle cell lipomatous tumor and suggesting a morphologic spectrum (atypical spindle cell/pleomorphic lipomatous tumor). Am J Surg Pathol. 2017, 41:1443-1455. 10.1097/PAS.0000000000000936

8. McCarthy AJ, Chetty R: Tumours composed of fat are no longer a simple diagnosis: an overview of fatty tumours with a spindle cell component. J Clin Pathol. 2018, 71:483-492. 10.1136/jclinpath-2017-204975

9. Anderson WJ, Fletcher CD, Jo VY: Atypical pleomorphic lipomatous tumor: expanding our current understanding in a clinicopathologic analysis of 64 cases. Am J Surg Pathol. 2021, 45:1282-1292. 10.1097/PAS.0000000000001706

10. Creytens D, Mentzel T, Ferdinande L, van Gorp J, Van Dorpe J, Flucke U: "Fat-rich" (spindle cell-poor) variant of atypical spindle cell/pleomorphic lipomatous tumor: striking mimic of "Classical" atypical lipomatous tumor/well-differentiated liposarcoma. Int J Surg Pathol. 2019, 27:868-871. 10.1177/1066896919846561

11. Boyd AS: An atypical pleomorphic lipomatous tumor arising on the cheek . J Cutan Pathol. 2019, 46:942-944. 10.1111/cup.13540

12. Wehrle CJ, Daigle JW, Ullah A, Sharma S, Ritter EF, Kruse EJ: Atypical spindle cell lipomatous lesion resected from patient with history of CLL. Am Surg. 2020, 86:1208-1211. 10.1177/0003134820933601

13. Yoshida Y, Nakabayashi M, Harada Y, Shingu T, Takubo K: A case report of atypical spindle cell lipomatous tumor of the tongue. Yonago Acta Med. 2020, 63:223-227. 10.33160/yam.2020.08.008

14. Berg SH, Massoud CM, Jackson-Cook C, Boikos SA, Smith SC, Mochel MC: A reappraisal of superficial pleomorphic liposarcoma: exploring the relationship to atypical spindle cell/pleomorphic lipomatous tumor. Am J Clin Pathol. 2020, 154:353-361. 10.1093/ajcp/aqaa045

15. Memon R, Abdelfatah MM, Patel C, et al.: Atypical spindle cell/pleomorphic lipomatous tumor of the stomach: A case report. Hum Pathol: Case Reps. 2021, 25:200540. 10.1016/j.ehpc.2021.200540

16. Hirsiger S, Dawson H, Grobbelaar AO, Vögelin E: Atypical pleomorphic lipomatous tumor of the right hand mimicing venous malformation. J Hand Surg Am. 2021, 10.1016/j.jhsa.2021.04.015

17. Bansal A, Goyal S, Goyal A, Jana M: WHO classification of soft tissue tumours 2020: an update and simplified approach for radiologists. Eur J Radiol. 2021, 143:109937. 10.1016/j.ejrad.2021.109937 


\section{Cureus}

18. Johnson CJ, Pynsent PB, Grimer RJ: Clinical features of soft tissue sarcomas . Ann R Coll Surg Engl. 2001, 83:203-205.

19. Strauss DC, Qureshi YA, Hayes AJ, Thway K, Fisher C, Thomas JM: The role of core needle biopsy in the diagnosis of suspected soft tissue tumours. J Surg Oncol. 2010, 102:523-529. 10.1002/jso.21600

20. Libbrecht S, Van Dorpe J, Creytens D: The rapidly expanding group of RB1-deleted soft tissue tumors: an updated review. Diagnostics (Basel). 2021, 11:430. 10.3390/diagnostics11030430 